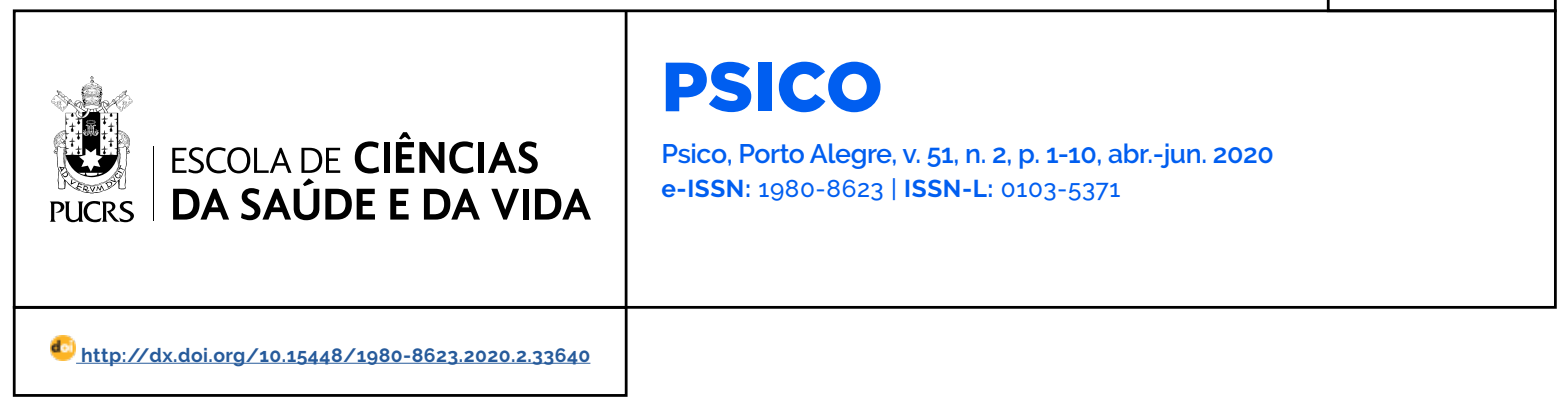

SEÇÃO: ARTIGO

\title{
Avaliação psicológica de pacientes após a alta da unidade de terapia intensiva
}

\author{
Psychological evaluation of patients after discharge from the intensive care unit \\ Evaluación psicológica de pacientes después de la alta de la unidad de terapia intensiva
}

\author{
Cristiane Rodrigues \\ Lopes $^{1}$ \\ orcid.org/0000-0003-3746-1540 \\ cris_rodriguees@hotmail.com
}

\section{Jaquilene Barreto da \\ Costa ${ }^{1}$ \\ orcid.org/0000-0002-0998-8559 \\ jaquihuop@yahoo.com.br}

Lukas Borges lepsen ${ }^{2}$ orcid.org/0000-0003-1539-9299

lukasiepsen@gmail.com

Recebido em: 21 mar. 2019. Aprovado em: 26 nov. 2019. Publicado em: 4 set. 2020.

\section{(c) (i)}

Artigo está licenciado sob forma de uma licença Creative Commons Atribuição 4.0 Internacional.
Resumo: A maioria dos pacientes sobrevive ao episódio de doença crítica, contudo, muitos deles podem desenvolver alterações psicológicas após a alta da UTI. Dada a natureza dos cuidados intensivos e as condições clínicas da maioria dos pacientes esta pesquisa tem como objetivo descrever os resultados da avaliação psicológica de pacientes três meses após a alta dos cuidados críticos. Foram avaliados 160 pacientes, destes 137 conseguiram responder à avaliação psicológica e os demais não foram avaliados em decorrência de algum comprometimento neurológico. Os instrumentos utilizados foram: planilha para coleta de dados do prontuário, instrumento de avaliação de memórias de UTI, Impact of Event Scale-Revised (IES-R) e Escala Hospitalar de Ansiedade e Depressão (HADS). Os resultados revelaram que a identificação precoce das complicações inerentes ao tratamento crítico poderá trazer benefícios para a prevenção de alterações emocionais/físicas crônicas subsequentes.

Palavras-chave: unidades de terapia intensiva, cuidados criticos, transtornos mentais, memória, psicologia.

Abstract: Most patients survive the critical illness episode, however, many of them may develop psychological changes after discharge from the ICU. Given the nature of intensive care and the clinical conditions of most patients this study aims to describe the results of the psychological evaluation of patients three months after discharge from critical care. 160 patients were evaluated, of these 137 were able to respond to psychological assessment and the rest were not evaluated due to some neurological impairment. The instruments used were: Worksheet for collecting data from the medical record, ICU memory evaluation tool, Impact of Event Scale-Revised (IES-R) and Hospital Anxiety and Depression Scale (HADS). The results revealed that the early identification of the complications inherent to the critical treatment may bring benefits to the prevention of subsequent chronic emotional / physical changes. Keywords: intensive care units, critical care, mental disorders, memory, psychology.

Resumen: La mayoria de los pacientes sobrevive al episodio de enfermedad critica, sin embargo, muchos de ellos pueden desarrollar alteraciones psicológicas después del alta de la UCl. Dada la naturaleza de los cuidados intensivos y las condiciones clínicas de la mayoria de los pacientes esta investigación tiene como objetivo describir los resultados de la evaluación psicológica de pacientes tres meses después del alta de los cuidados críticos. Se evaluaron 160 pacientes, de estos 137 pudieron responder a la evaluación psicológica y el resto no se evaluó debido a algún deterioro neurológico. Los instrumentos utilizados fueron: Planilla para la recolección de datos del prontuario, instrumento de evaluación de memorias de UTI, Impact of Event Scale-Revised (IES-R) y Escala Hospitalaria de Ansiedad y Depresión (HADS). Los resultados revelaron que la identificación precoz de las complicaciones inherentes al tratamiento crítico puede traer beneficios para la prevención de alteraciones emocionales / físicas crónicas subsiguientes. Palabras clave: unidades de terapia intensiva, cuidados críticos, trastornos mentales, memoria, psicología. 
A hospitalização na Unidade de Terapia Intensiva (UTI) pode representar uma situação em que muitos pacientes estão psicologicamente vulneráveis. Essa fragilidade está relacionada a vários fatores, como risco real ou potencial de morte, procedimentos invasivos, gravidade da doença, além das condições extremas associadas à hospitalização (Chahraoui, Laurent, Bioy, \& Quenot, 2015).

A maioria dos pacientes sobrevive ao episódio de doença crítica, contudo, até 30\% podem sofrer de problemas psicológicos devido à exposição a muitos estressores durante a internação na UTI. Tal exposição pode interferir no estado emocional do paciente e no desencadeamento de alterações psicológicas após a alta. Transtornos mentais após a alta da UTI incluem transtorno de estresse pós-traumático (TEPT), ansiedade e depressão (Milton, Brück, Schandl, Bottai, \& Sackey, 2017).

O TEPT manifesta-se por meio de um conjunto de reações emocionais e comportamentais; seus sintomas causam sofrimento significativo em diversas áreas de funcionamento, como isolamento social e embotamento afetivo. Além disso, sua sintomatologia frequentemente está associada às comorbidades psiquiátricas, como os transtornos de ansiedade, depressão, transtorno de pânico e dissociação. Os fatores de risco associados ao desenvolvimento do TEPT pós-UTI incluem pacientes que experimentaram condições de estresse agudo, medo, regressão, confusão e agitação durante a internação (Chahraoui et al., 2015). Outros estudos também identificaram diferentes fatores de risco para TEPT, incluindo histórico de ansiedade ou depressão, doses elevadas de sedação com benzodiazepínicos, tempo de sedação, memórias de experiências assustadoras ou psicóticas durante a permanência na UTI, mulheres, pacientes com idade abaixo de 50 anos, duração da ventilação mecânica, tempo de permanência, agitação durante a internação e contenção física (Wade et al., 2012; Costa et al., 2014).

Em relação à presença de sintomas de ansiedade e de depressão pós-alta da UTI, no estudo de Nikayin et al. (2016), a prevalência de sintomas de ansiedade, usando um ponto de corte de $\geq 8$ na escala HADS, foi de $32 \%$ aos 2-3 meses. Histórico de transtorno psiquiátrico prévio e memórias de experiências delirantes na UTI foram potenciais fatores de risco para ansiedade. Já Sintomas de depressão ocorrem em cerca de $29 \%$ dos sobreviventes, podendo aparecer em três, seis e até um ano após a alta da UTI. O uso de benzodiazepínicos na UTI foi o principal fator de risco clínico para depressão. Além disso, a posição socioeconômica e o histórico psicológico foram indicados como fator de risco independentes para depressão (Wade et al., 2012). Em um estudo recente identificou-se que sintomas psicológicos prévios, assim como a presença de sintomas de sofrimento psíquico na UTI ou hospital, incluindo raiva, nervosismo e sintomas de estresse agudo estão fortemente associados a sintomas depressivos após a alta. (Milton, Brück, Schandl, Bottai, \& Sackey, 2017).

Um estudo brasileiro mostrou que até $40 \%$ dos sobreviventes de UTI necessitam de reinternação e $52 \%$ visitaram o departamento de emergência durante o primeiro ano pós-internação (Ranzani, Zampieri, Besen, Azevedo, \& Park, 2015). Um outro estudo mostrou que $21 \%$ dos pacientes que receberam alta da UTI, morreram no ano seguinte (Herridge et al., 2016). Isso confirma a necessidade de acompanhamento ambulatorial, no entanto, poucos serviços acompanham seus pacientes após a alta da UTI, e o número de serviços de reabilitação voltados para a recuperação é escasso. Portanto, verifica-se a necessidade de expansão do cuidado mais abrangente, com a inclusão do acompanhamento por equipe multiprofissional do paciente após a alta. A relevância em compreender e detectar precocemente as consequências psicológicas após a alta da UTI tem sido demonstrada pelos dados mencionados na literatura, bem como a necessidade de reunir subsídios para uma compreensão ampliada da recuperação do paciente. Desta forma, o presente estudo tem como objetivo descrever os resultados da avaliação psicológica três meses após a alta dos cuidados críticos.

\section{Método}

Trata-se de um estudo prospectivo longitudinal, com abordagem quantitativa, realizado na UTI de 
um hospital-escola localizado na região Oeste do Paraná, Brasil. A UTI em estudo é referência de alta complexidade para 26 municípios da região e conta com 15 leitos destinados a pacientes adultos acometidos de patologias clínicas diversas. Participaram do estudo todos os pacientes que retornaram na consulta ambulatorial no periodo de fevereiro a dezembro de 2017, que preencherem os seguintes critérios de inclusão: maiores de 18 anos e que consentiram sua participação na pesquisa.

A primeira etapa de coleta de dados ocorreu durante o periodo da internação do paciente na UTI. Para tanto, realizou-se diariamente o rastreamento das admissões e das altas de pacientes por meio da verificação do livro de registro da unidade. Foram coletados, a partir da consulta ao prontuário, telefone de contato do paciente, dados sociodemográficos e clínicos.

A avaliação ocorreu três meses após a alta da UTI por ocasião do retorno do paciente no Ambulatório Interdisciplinar de Seguimento em Terapia Intensiva da instituição pesquisada. Nessa etapa, foram aplicados os instrumentos para avaliar as recordações do paciente durante sua permanência na UTI, sintomas de ansiedade, depressão e TEPT.

\section{Instrumentos}

Planilha para coleta de dados do prontuário. Nesta planilha foram registrados dados sociodemográficos (nome, idade, sexo, escolaridade, estado civil) e clínicos (tempo de Ventilação Mecânica (VM), de permanência na UTI e enfermaria, causa de admissão e complicações na UTI).

Instrumento de avaliação de memórias de UTI (Costa \& Marcon, 2009). O referido instrumento é do tipo checklist constituido por duas questões abertas e nove itens agrupados em quatro grandes domínios: memórias relacionadas ao tratamento (tubo no nariz e boca, extubação, aspiração, entre outros); memórias do ambiente (visita da familia, barulho, alta para enfermaria, entre outros); memórias relacionadas às experiências emocionais (dor, sede, fome, agitação, confusão, medo, entre outros) e àquelas relacionadas às memórias ilusórias (sonhos, pesadelos e alucinações). Para identificação dos tipos de memórias, classificou- se como apresentando amnésia total - aqueles pacientes que não tiveram nenhuma recordação do período de internação na UTI; memórias de fatos reais - aqueles com recordações relacionadas a fatos reais referentes ao tratamento, ao ambiente e eventos emocionais; e, por fim, memórias ilusórias - relacionadas a eventos irreais, como pesadelos, sonhos e alucinações:

Impact of Event Scale-Revised (IES-R) (Weiss \& Marmar, 1997). A escala contém 22 itens agrupados em três subescalas. A primeira subescala está relacionada com sintomas de intrusão (sete itens), caracterizada pela presença de pensamentos e de imagens indesejadas. A segunda é caracterizada pela presença de pensamentos de evitação (oito itens). E, por último, a subescala de hiperestimulação (sete itens), que inclui distúrbios do sono, sintomas de flashback e labilidade emocional. As respostas são pontuadas em uma escala tipo Likert de cinco pontos e o seu escore varia de o a 66 pontos. Um escore > 20 indica a presença de sintomas de TEPT e uma pontuação $\geq 33$ indica provável transtorno clínico (Creamer, Bell, \& Failla, 2003). A IES-R tem demonstrado boa consistência interna, validade preditiva e discriminativa (Caiuby, 2012).

Para avaliação de sintomas de ansiedade e depressão foi utilizado a escala Hospitalar de Ansiedade e Depressão (HADS) que possui 14 itens, sendo sete voltados para a avaliação da ansiedade (HADS-A) e sete para a depressão (HADS-D). Cada um dos seus itens pode ser pontuado de zero a três, compondo uma pontuação máxima de 21 pontos para cada escala. Foram adotados os pontos de cortes apontados por Zigmond e Snaith: um escore acima de $\geq 9$ para ambas as subescalas indica sintomas de ansiedade e de depressão (Marcolino, Suzuki, Alli, Gozzani, \& Mathias, 2007).

\section{Procedimentos éticos e de coleta dos dados}

O estudo foi desenvolvido em conformidade com o preconizado pela Resolução 510/2016 do Conselho Nacional de Saúde (CNS) acerca dos cuidados éticos para pesquisas com seres humanos. O projeto foi apreciado pelo Comitê de Ética em Pesquisa da Universidade Estadual do Oeste do 
Paraná (Unioeste) sob o CAAE 0022.0.276.000-09.

$A$ avaliação de todos os pacientes incluidos no estudo ocorreu após a assinatura do Termo de Consentimento Livre e Esclarecido (TCLE).

\section{Análise estatística}

Os dados foram armazenados no programa Microsoft Excel $®$ e para análise e interpretação dos resultados foram utilizados os programas IBM SPSS Statistics 22 e Python 3.6.4 usando a biblioteca Pandas 0.22.0. Para comparar os grupos com alterações psicológicas, as seguintes variáveis foram categorizadas como independentes: sexo, idade, religião, causa de admissão, tempo de UTI, tempo de ventilação mecânica, recordações de UTI e tipos de memórias. O Teste Exato de Fisher foi empregado para investigar a associação de cada fator com o desfecho, usando um nível de significância de 5\%. Estatísticas descritivas foram realizadas para todas as variáveis, visando à caracterização geral dos participantes e à descrição da presença de recordações referentes à UTI. (Tabela 1)

Tabela 1 - Valores referentes ao teste estatistico das alterações psicológicas (Tept, depressão e ansiedade)

\begin{tabular}{|c|c|c|c|}
\hline Alteraçōes Psicológicas & Positivo & Negativo & $\mathrm{p}$ \\
\hline Sexo & & & 0.017 \\
\hline Masculino & 7 & 83 & \\
\hline Feminino & 11 & 37 & \\
\hline Idade & & & 1.0 \\
\hline $47+$ & 8 & 50 & \\
\hline 47- & 10 & 70 & \\
\hline Religiăo & & & 0.192 \\
\hline Católico & 9 & 80 & \\
\hline Protestante & 9 & 40 & \\
\hline Tempo de intemação UTI & & & 0.063 \\
\hline $10+$ & 10 & 38 & \\
\hline $10-$ & 8 & 82 & \\
\hline Memória UTI & & & 0.621 \\
\hline Sim & 7 & 55 & \\
\hline Não & 11 & 65 & \\
\hline Tempo de VM & & & 0.009 \\
\hline $6+$ & 12 & 40 & \\
\hline 6- & 6 & 79 & \\
\hline
\end{tabular}

\section{Resultados}

Ao todo, 407 pacientes foram de alta da UTI durante o período do estudo. Desses, 160 atenderam aos critérios de inclusão e retornaram na consulta ambulatorial e 137 foram avaliados pela equipe de psicologia no seguimento três meses após a alta da UTI. Os demais pacientes deixaram de ser avaliados em função de sequelas neurológicas, dificuldade de concluir avaliação, bem como dificuldade para responder, conforme demonstrado na Figura 1..

Figura 1 - Fluxograma dos Pacientes Admitidos na Unidade de Terapia Intensiva Durante o Periodo do Estudo

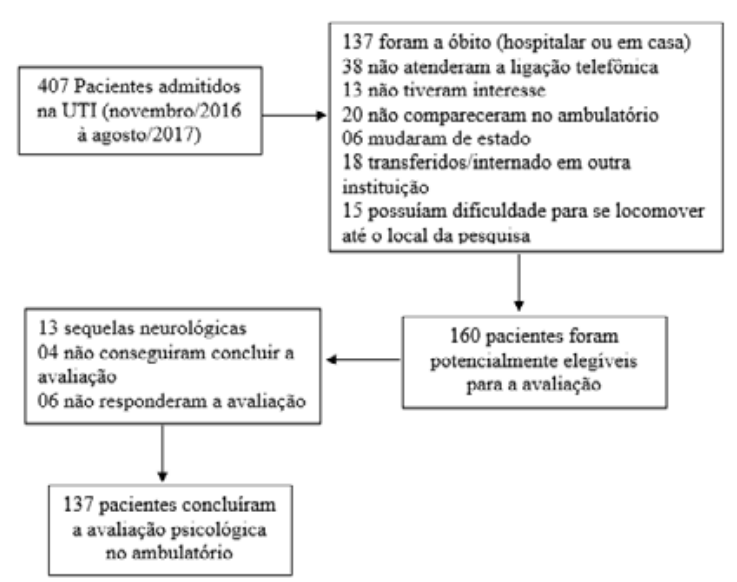

Dos pacientes avaliados, 102 (63.7\%) eram do sexo masculino, a idade variou entre 17 a 87 anos, com uma média de 48 anos ( \pm 17.3 ). Quanto à religião, 99 (81,1\%) se autodeclararam católicos, $22(18,0 \%)$ evangélicos e $1(0,8 \%)$ adventistas. Metade dos pacientes 81 (50,6\%) eram casados; 48 (30\%) solteiros; 14 (11,7\%) divorciados; 10 (8,3\%) viúvos e 7 (4,4\%) não declarado.

A maioria dos pacientes 57 (35,6\%) foi admitido por motivo clínico, seguidos daqueles por motivo neurológico não trauma 37 (23,1\%), neurológico trauma $34(21,2 \%)$ e cirúrgico $32(20 \%)$. O tempo médio de permanência na UTI foi de 10 dias $( \pm$ 7.5), variando de 1 a 44 dias e a grande maioria 124 (90,5\%) permaneceu em ventilação mecânica, com um tempo médio de 7,6 dias $( \pm 7,6)$. A média de APACHE II foi de 19,8 \pm 7,7. 
Tabela 2 - Características Demográficas e Clínicas dos Pacientes Avaliados ( $n=137)$

\begin{tabular}{|c|c|c|}
\hline Variáveis & n $(\%)$ & Média/Desvio Padrão \\
\hline \multicolumn{3}{|l|}{ Sexo } \\
\hline Masculino & $102(63,7)$ & \\
\hline Feminino & $58(36,2)$ & \\
\hline Faixa etária & & $48( \pm 17,3)$ \\
\hline $18-40$ & $58(36,3)$ & \\
\hline $41-60$ & $60(37,5)$ & \\
\hline$>60$ & $41(25,6)$ & \\
\hline \multicolumn{3}{|l|}{ Causa admissão } \\
\hline Trauma & $34(21,3)$ & \\
\hline Clínica & $57(35,6)$ & \\
\hline Cirúrgica & $32(20)$ & \\
\hline Neurológica & $37(23,1)$ & \\
\hline \multicolumn{3}{|l|}{ Sequelas pós UTI } \\
\hline Sim & $86(53,7)$ & \\
\hline Não & $74(46,2)$ & \\
\hline Tempo de UTI & & $10( \pm 7,5)$ \\
\hline Até $48 \mathrm{hs}$ & $11(6,9)$ & \\
\hline $3-7$ dias & $67(41,9)$ & \\
\hline$>7$ dias & $81(50,6)$ & \\
\hline Tempo V.M. & & $7,6( \pm 7,6)$ \\
\hline$\leq 2$ dias & $38(23,7)$ & \\
\hline$>2$ dias & $104(65)$ & \\
\hline Tempo internação pós-UTI & & $25( \pm 18,7)$ \\
\hline Até 7 dias & $10(6,25)$ & \\
\hline 8-14 dias & $38(23,75)$ & \\
\hline$>14$ dias & $111(69,4)$ & \\
\hline APACHE II & & $19,8( \pm 7,7)$ \\
\hline
\end{tabular}

Na avaliação de seguimento três meses após a alta da UTI, 14,37\% dos pacientes apresentaram alterações psicológicas. Desses, 6,56\% pacientes tinham sintomas de depressão; 2,91\% sintomas de ansiedade e 4,37\% apresentaram sintomatologia para TEPT. A média de idade dos pacientes que sofreram alterações psicológicas foi de 46.2 ( \pm 14.7). Do total de pacientes avaliados, 3,64\% apresentaram morbidade psicológica.

A presença de alteração psicológica foi mais comum em pacientes mulheres, com média de idade de 47 anos, que permaneceram internadas por um periodo médio de 12,63 dias na UTI e que permaneceram em média 7.9 dias em ventilação mecânica. Em nosso estudo, de nove pacientes com sintomas de ansiedade, quatro eram mulheres, com idade entre 37 e 65 anos.

Quanto à presença do TEPT, seis (4,37\%) dos pacientes apresentaram sintomas de TEPT quando foi utilizado um ponto de corte $>20$. Para melhor precisão diagnóstica, foi utilizado também o ponto de corte $\geq 33$ pontos e, nesse caso, somente um paciente $(0,72 \%)$ apresentou o transtorno clínico, confirmado com dados da entrevista psicológica realizada no ambulatório. Dos seis pacientes com TEPT, cinco eram mulheres com uma média de permanência de 13,6 dias na UTI e 35,1 dias de internação hospitalar e que permaneceram em ventilação mecânica por mais de dois dias. Desses pacientes, três tiveram memórias ilusórias. 
A Tabela 3 representa um comparativo entre pacientes com e sem sintomas de TEPT.

Tabela 3 - Comparação dos pacientes com presença de TEPT e pacientes sem TEPT

\begin{tabular}{lcc}
\hline & com TEPT & sem TEPT \\
\hline Tempo VM & $13.40( \pm 6.80)$ & $6.98( \pm 7.69)$ \\
Internação UTI & $13.67( \pm 6.89)$ & $9.53( \pm 7.46)$ \\
Internação Hospitalar & $35.17( \pm 23.83)$ & $23.18( \pm 16.82)$ \\
Sexo & & \\
Masculino & 1 & 89 \\
Feminino & 5 & 43 \\
Memória da UTI & & \\
Sim & 4 & 58 \\
Não & 2 & 74 \\
\hline
\end{tabular}

A maioria dos pacientes $(60,7 \%)$ não apresentou nenhuma memória do periodo de internação na UTI. Dos que se recordam uma grande proporção (63\%) relatou memórias de fatos reais, seguido por 29\% com memórias de fatos reais e ilusórias, e $8 \%$ com memórias ilusórias, como sonhos, pesadelos e alucinações.
Quanto às recordações de fatos reais, as lembranças mais frequentes foram: alta da UTI para enfermaria (91,22\%), visita dos familiares (91,22\%), higiene/banho (87,71\%), a sonda vesical $(80,70 \%)$, e sentir fome/sede $(68,42)$. Para uma melhor avaliação das recordações do paciente na UTI foi feita uma pergunta aberta referente à experiência mais desagradável durante a internação do paciente na UTI. Um número elevado de pacientes (70,2\%), não se recordou de quaisquer experiências desagradáveis. Os demais relataram memórias ruins relacionadas ao tratamento (14.5\%), experiências pessoais ( $8 \%$ ) e em relação ao ambiente $(6,5 \%)$

Referente às sequelas pós-UTI, observouse que $46.2 \%$ pacientes apresentaram algum tipo de sequela. Desses, 47,3\% tinham sequela física e $44,6 \%$ sequela neurológica. Durante o atendimento ambulatorial houve a necessidade de referenciar estes pacientes para as seguintes especialidades: $13,7 \%$ foram encaminhados para fisioterapia, seguido de $11,2 \%$ para a psicologia, $6,2 \%$ para psiquiatria e 3,12\% para neurologia.

Tabela 4 - Resultados das Avaliações Realizadas de Acordo com os Instrumentos Utilizados (HADS, Memória de UTI, IES)

\begin{tabular}{|l|c|c|c|c}
\hline Instrumentos & N & Média/DP & Variação \\
\hline HADS (n=138) & & & & \\
\hline Ansiedade & 9 & 6,5 & $12,1( \pm 1,8)$ & $9-15$ \\
\hline Depressão & 9 & 6,9 & $11,8( \pm 3,2)$ & $9-17$ \\
\hline Ansiedade/depressão & 4 & 2,9 & $10,3 / 14( \pm 1,23,6)$ & $9 / 10-11 / 17$ \\
\hline Memória & & & & \\
\hline Sim & 62 & 38,7 & & \\
\hline Não & 98 & 61,3 & & \\
\hline Tipos de memórias (n=62) & & & & \\
\hline Fatos reais & 39 & 62,9 & & \\
\hline Ilusórias & 5 & 8 & & \\
\hline Reais e ilusórias & 18 & 29 & & \\
\hline Experiência desagradável UTI(n=138) & & & & \\
\hline Nenhuma & 97 & 70,2 & & \\
\hline Tratamento & 20 & 14,5 & & \\
\hline Ambiente & 9 & 6,5 & & \\
\hline Exp. pessoais & 11 & 8 & & \\
\hline Outros & 1 & 0,7 & & \\
\hline IES & & & \\
\hline$>20$ & 5 & 3,64 & & \\
\hline$>33$ & 1 & 0,72 & \\
\hline
\end{tabular}




\section{Discussão}

Neste estudo, pretendeu-se compreender as experiências psicológicas de pacientes críticos e identificar possiveis alterações psicológicas três meses após a alta da UTI. Observou-se que para muitos pacientes, a doença crítica pode significar um evento traumático e representa uma ameaça à vida estando associada a uma variedade de estressores comumente presentes no ambiente da UTI (Long, Kross, Davydow, \& Curtis, 2014).

A prevalência de alterações psicológicas após cuidados críticos é alta e tais sintomas parecem persistir ao longo do tempo, assim, os pacientes apresentam diferentes cursos no desencadeamento dos sintomas, podendo persistir por periodo prolongado (Davydow, Gifford, Desai, Needham, \& Bienvenu, 2008). Uma destas alterações é o estresse pós-traumático, que é descrita como uma condição clínica desencadeada pela experiência de um evento traumático com risco de morte (ou percebido como risco de morte), que causa fortes respostas subjetivas, como recordações intrusivas do evento, sintomas de hiperexcitação e comportamento evitativo relacionado ao evento traumático (APA, 2013).

Chahraoui (2015), em um estudo que avaliou alterações psicológicas três meses após a permanência na Unidade de Terapia Intensiva constatou que $15 \%$ dos pacientes apresentaram transtorno de estresse pós-traumático. Em um outro estudo recente foi encontrada prevalência entre 3.70 e $43.73 \%$, sendo $15,93 \%$ aos 3 meses pós-alta da UTI (Righy et al., 2019). Um estudo longitudinal relatou que a prevalência de TEPT e de sintomas depressivos foi de $16 \%$ e $31 \%$, respectivamente, aos 3 meses pós-UTI (Davydow, Zatzick, Hough, \& Katon, 2013).

No presente trabalho apenas cinco pacientes (3,64\%) apresentaram sintomas de TEPT quando foi utilizado um ponto de corte $>20$ e utilizando o ponto de corte $\geq 33$ pontos, somente 1 paciente $(0,72 \%)$ apresentou o transtorno clínico. A diferença observada na baixa incidência de pacientes com transtorno, em comparação com estudos anteriormente citados que encontraram alta incidência, pode ser explicada pelo fato de ter sido utilizado para a avaliação não só o instrumento IES-R, mas também dados da entrevista psicológica, bem como devido ao fato de esse evento ter sido estudado na literatura em periodos distintos, com diferentes metodologias e coortes de pacientes (Davydow, Zatzick, Hough, \& Katon, 2013; Chahraoui, 2015; Righy et al., 2019).

Em um estudo realizado com 62 pacientes atendidos nos pós-UTI, O TEPT também foi raro, ocorrendo em apenas três dos pacientes testados (5\%) (Sevin et al., 2018). É necessário considerar que a perda do seguimento pode ser particularmente problemática na avaliação desse transtorno, uma vez que o comportamento de esquiva, caracteristica fundamental do TEPT, pode fazer com que alguns sobreviventes da UTI evitem participar e retornar em ambulatórios de follow-up. Por esse motivo segundo Long, Kross, Davydow \& Curtis (2014), muitos dos estudos citados podem subestimar a verdadeira prevalência de TEPT.

Mulheres e pacientes jovens têm sido indicados como potenciais fatores de risco para desenvolver sintomas de estresse pós-traumático após uma doença critica, porém esses resultados ainda são inconsistentes entre os estudos (Wade, Hardy, Howell, \& Mythen, 2013). No entanto, esse fator corrobora com o resultado do presente estudo, pois dentre os seis pacientes que apresentaram sintomas de TEPT, cinco eram mulheres. Do total de pacientes com TEPT cinco permaneceram em VM, com uma média de 13,6 dias na UTI e 35,1 dias de internação hospitalar. Desses pacientes, três tiveram memórias ilusórias.

Já em relação à ansiedade, estudos realizados com a população geral têm mostrado que esse transtorno é $60 \%$ maior em mulheres, com mais prevalência entre o grupo de 30 e 44 anos (Kessler et al., 2005). Em nosso estudo, de nove pacientes com sintomas de ansiedade, quatro eram mulheres, com idade entre 37 e 65 anos de idade.

Em um estudo de Schandl et al. (2011), 66\% dos sobreviventes da UTI apresentavam funções fisicas prejudicadas. Isso é afirmado no presente estudo quando se verifica a necessidade de encaminhamento e de acompanhamento após a alta. No nosso estudo, um terço dos pacientes foram encaminhados para outras especialidades (fisioterapia $14,4 \%$, seguido de $11,2 \%$ para a 
psicologia e 6,9\% para psiquiatria).

Sequelas físicas e neurológicas foram observadas no presente estudo. Um total de $46,25 \%$ dos pacientes apresentou sequelas, sendo que $47,3 \%$ apresentaram sequela física e 44,6\% danos neurológicos. Um terço dos pacientes foram encaminhados para outras especialidades (fisioterapia $14,4 \%$, seguido de $11,2 \%$ para a psicologia e 6.9\% para psiquiatria), havendo a necessidade de acompanhamento após a alta da UTI.

Em um estudo de Schandl et al. (2011), 66\% dos sobreviventes da UTI apresentavam também funções fisicas prejudicadas. A função física tem um grande impacto na qualidade de vida do paciente após a alta da UTI, interferindo diretamente nas atividades laborais. Em um estudo de 136 pacientes após a alta da UTI, 65\% destes não estavam trabalhando em comparação com 40\% antes da admissão na UTI (Kvåle, Ulvik, Flaatten, 2003).

Quando comparada a distribuição dos pacientes avaliados por sexo, verificou-se que houve predominância de pacientes de sexo masculino (63.7\%). Estudos brasileiros vêm confirmando tal achado, com resultados que variaram de $53 \%$ a $64 \%$, mostrando que mais de 50\% dos internados são do sexo masculino (de Albuquerque, 2017; Hernández Rodriguez et al., 2016; Melo, Menegueti, \& Laus, 2014). Esse dado pode ser resultante do baixo interesse pela saúde, tendo em vista que em sua maioria quando acontece a adesão por parte deles, muitas vezes a gravidade já está estabelecida.

\section{Conclusão}

O estudo demonstrou que para alguns pacientes a internação em UTI pode se constituir em um evento traumático podendo desencadear alterações psicológicas em curto prazo. Constatou-se também que, além de alterações psicológicas, as complicações físicas três meses após a alta também estiveram presentes, havendo a necessidade de referenciar o paciente para outras especialidades. Esse conhecimento demanda a necessidade de ambulatórios de follow-up, embora poucos serviços acompanhem o paciente após a alta da UTI.
Portanto, a identificação precoce das complicações inerentes ao tratamento crítico poderá trazer benefícios, tanto para a reabilitação física do paciente quanto para a prevenção de alterações emocionais/físicas crônicas subsequentes. Portanto, o conhecimento de equipes de saúde na identificação dessa condição e no desenvolvimento de estratégias de intervenção é recomendado. Uma das limitações do estudo foi que os dados existentes não esclarecem se as alterações psicológicas são o resultado da doença critica, ou se as alterações pós-UTI refletem a condição clínica prévia do paciente.

\section{Conflitos de interesse}

Os autores declaram não existir quaisquer conflitos de interesse.

\section{Referências}

Associação Americana de Psiquiatria. (2013). Manual diagnóstico e estatístico de transtornos mentais. BMC Med. 17, 133-137. (DOI INEXISTENTE)

Caiuby, A. V. S., Lacerda, S. S., Quintana, M. I., Torii, T. S., \& Andreoli, S. B. (2012). Adaptação transcultural da versão brasileira da Escala do Impacto do Evento - Revisada (IES-R). Cadernos de Saúde Pública, 28(3), 597-603. https://dx.doi.org/10.1590/ $\underline{\text { S0102-311X2012000300019 }}$

Chahraoui, K., Laurent, A., Bioy, A., \& Quenot, J. P. (2015). Psychological experience of patients 3 months after a stay in the intensive care unit: A descriptive and qualitative study. Journal of critical care, 30(3), 599-605. https://doi.org/10.1016/j.jcrc.2015.02.016

Costa, J. B. D., \& Marcon, S. S. (2009). Elaboração e avaliação de um instrumento para identificar memórias referentes à unidade de terapia intensiva. $J$ Bras Psiquiatr, 58(4), 223-30. https://doi.org/10.1590/ s0047-20852009000400002

Costa, J. B. D., Marcon, S. S., Macedo, C. R. L. D., Jorge, A. C., \& Duarte, P. A. D. (2014). Sedação e memórias de pacientes submetidos à ventilação mecânica em unidade de terapia intensiva. Revista Brasileira de Terapia Intensiva, 26(2), 122-129. https:// doi.org/10.5935/0103-507×.20140018

Creamer, M., Bell, R., \& Failla, S. (2003). Psychometric properties of the impact of event scale-revised. Behaviour research and therapy, 41(12), 1489-1496. https://doi.org/10.1016/j.brat.2003.07.010

Davydow, D. S., Gifford, J. M., Desai, S. V., Needham, D. M., \& Bienvenu, O. J. (2008). Posttraumatic stress disorder in general intensive care unit survivors: a systematic review. General hospital psychiatry, 30(5), 421-434. https://doi.org/10.1016/j.genhosppsych.2008.05.006 
Davydow, D. S., Zatzick, D., Hough, C. L., \& Katon, W. J. (2013). A longitudinal investigation of posttraumatic stress and depressive symptoms over the course of the year following medical-surgical intensive care unit admission. General hospital psychiatry, 35(3), 226-232. https://doi.org/10.1016/i.genhosppsych.2012.12.005

de Albuquerque, J. M., da Silva, R. F. A., \& de Souza, R. F. F. (2017). perfil epidemiológico e seguimento após alta de pacientes internados em unidade de terapia intensiva. Cogitare Enfermagem, 22(3). https:// doi.org/10.5380/ce.v22i3.50609

Hernández Rodriguez, A., Camargo Bub, M. B., Perão, O. F., Zandonadi, G., \& Hernández Rodriguez, M. D. J. (2016). Caracteristicas epidemiológicas e causas de óbitos em pacientes internados em terapia intensiva. Revista Brasileira de Enfermagem, 69(2). https://doi. org/10.1590/0034-7167.2016690204i

Herridge, M. S., Chu, L. M., Matte, A., Tomlinson, G., Chan, L., Thomas, C., ... Ferguson, N. D. (2016). The RECOVER program: disability risk groups and 1-year outcome after 7 or more days of mechanical ventilation. American journal of respiratory and critical care medicine, 194(7), 831-844. https://doi.org/10.1164/ rccm.201512-23430c

Kapfhammer, H. P., Rothenhäusler, H. B., Krauseneck, T., Stoll, C., \& Schelling, G. (2004). Posttraumatic stress disorder and health-related quality of life in long-term survivors of acute respiratory distress syndrome. American Journal of Psychiatry, 161(1), 45-52. https://doi.org/10.1176/appi.ajp.161.1.45

Kessler, R. C., Berglund, P., Demler, O., Jin, R., Merikangas, K. R., \& Walters, E. E. (2005). Lifetime prevalence and age-of-onset distributions of DSM-IV disorders in the National Comorbidity Survey Replication. Archives of general psychiatry, 62(6), 593-602. https://doi.org/10.1001/archpsyc.62.6.593

Kvåle, R., Ulvik, A., \& Flaatten, H. (2003). Follow-up after intensive care: a single center study. Intensive care medicine, 29(12), 2149-2156. https://doi. org/10.1007/s00134-003-2034-2

Long, A. C., Kross, E. K., Davydow, D. S., \& Curtis, J. R. (2014). Posttraumatic stress disorder among survivors of critical illness: creation of a conceptual model addressing identification, prevention, and management. Intensive care medicine, 40(6), 820-829 https:// doi.org/10.1007/s00134-014-3306-8

Marcolino, J. A. M., Suzuki, F. M., Alli, L. A. C., Gozzani, J. L., \& Mathias, L. A. D. S. T. (2007). Medida da ansiedade e da depressão em pacientes no pré-operatório. Estudo comparativo. Rev Bras Anestesiol, 57(2), 157-166. https://doi.org/10.1590/s003470942007000200004

Melo, A. C. D. L., Menegueti, M. G., \& Laus, A. M. (2014). Perfil de pacientes de terapia intensiva: subsídios para a equipe de enfermagem. Revista de Enfermagem UFPE On Line, 8(9), 3142-3148. (DOI INEXISTENTE)

Milton, A., Brück, E., Schandl, A., Bottai, M., \& Sackey, P. (2017). Early psychological screening of intensive care unit survivors: a prospective cohort study. Critical Care, 21(1), 273. https://doi.org/10.1186/s13054-017-1813-Z
Nikayin, S., Rabiee, A., Hashem, M. D., Huang, M., Bienvenu, O. J., Turnbull, A. E., \& Needham, D. M. (2016). Anxiety symptoms in survivors of critical illness: a systematic review and meta-analysis. General hospital psychiatry, 43, 23-29. https://doi. org/10.1016/j.genhosppsych.2016.08.005

Ranzani, O. T., Zampieri, F. G., Besen, B. A., Azevedo, L. C., \& Park, M. (2015). One-year survival and resource use after critical illness: impact of organ failure and residual organ dysfunction in a cohort study in Brazil. Critical Care, 19(1), 269. https://doi. org/10.1186/s13054-015-0986-6

Schandl, A. R., Brattström, O. R., Svensson-Raskh, A., Hellgren, E. M., Falkenhav, M. D., \& Sackey, P. V. (2011). Screening and treatment of problems after intensive care: a descriptive study of multidisciplinary follow-up. Intensive and Critical Care Nursing, 27(2), 94-101. https://doi.org/10.1016/j.iccn.2011.01.006

Sevin, C. M., Bloom, S. L., Jackson, J. C., Wang, L., Ely, E. W., \& Stollings, J. L. (2018). Comprehensive care of ICU survivors: Development and implementation of an ICU recovery center. Journal of critical care, 46, 141-148. https://doi.org/10.1016/j.jcrc.2018.02.011

Stein, M. B., Walker, J. R., Hazen, A. L., \& Forde, D. R. (1997). Full and partial posttraumatic stress disorder: findings from a community survey. The American Journal of Psychiatry, 154(8), 1114. https://doi. org/10.1176/ajp.154.8.1114

Wade, D. M., Howell, D. C., Weinman, J. A., Hardy, R. J., Mythen, M. G., Brewin, C. R., ... Raine, R. A. (2012). Investigating risk factors for psychological morbidity three months after intensive care: a prospective cohort study. Critical Care, 16(5), R192. https://doi.org/10.1186/cc11677

Wade, D., Hardy, R., Howell, D., \& Mythen, M. (2013). Identifying clinical and acute psychological risk factors for PTSD after critical care: a systematic review. Minerva Anestesiol, 79(8), 944-63. (DOI INEXISTENTE)

Weiss, D. S., \& Marmar, C. R. (1997). The Impact of Event Scale-Revised In: Wilson, J.P., Keane, T.M., editors. Assessing psychological trauma and ptsd. (pp. 399-411). New York: Guilford. APA PsycTests. https:// doi.org/10.1037/t12199-000

\section{Cristiane Rodrigues Lopes}

Graduada em Psicologia pela Universidade Paranaense (UNIPAR, Cascavel, PR, Brasil). Atualmente é psicóloga residente do Hospital de Clínicas de Porto Alegre (HCPA).

\section{Jaquilene Barreto da Costa}

Mestre em Ciências da Saúde pela Universidade Estadual de Maringá (UEM), psicóloga efetiva do Hospital Universitário do Oeste do Paraná, professora do curso de residência em fisioterapia intensiva pela UNIOESTE, Cascavel-PR 
10/10 Psico, Porto Alegre, v. 51, n. 2, p. 1-10, abr.-jun. 2020 | e-33640

\section{Lukas Borges lepsen}

Graduando em Ciência da Computação pela Universidade Federal de Pelotas (UFPEL) e cientista de dados na Elixir Al

\section{Endereço para correspondência}

Jaquilene Barreto da Costa

Av. Tancredo Neves, 3224

Santo Onofre, 85806-470

Cascavel, PR, Brasil 